\title{
La religion dans les décisions relatives à la santé et à l'éducation des enfants en droit canadien et québécois
}

José Woehrling

\section{(Q) OpenEdition}

Journals

Édition électronique

URL : http://journals.openedition.org/rdr/881

DOI : $10.4000 /$ rdr.881

ISSN : 2534-7462

\section{Éditeur}

Presses universitaires de Strasbourg

Édition imprimée

Date de publication : 3 mai 2017

Pagination : 157-174

ISBN : 978-2-86820-973-3

ISSN : 2493-8637

\section{Référence électronique}

José Woehrling, «La religion dans les décisions relatives à la santé et à l'éducation des enfants en droit canadien et québécois », Revue du droit des religions [En ligne], 3 | 2017, mis en ligne le 03 février 2020, consulté le 10 décembre 2020. URL : http://journals.openedition.org/rdr/881 ; DOI : https:// doi.org/10.4000/rdr.881

\section{(c) (†) (8)}

La revue du droit des religions est mise à disposition selon les termes de la Creative Commons Attribution - Pas d'Utilisation Commerciale 4.0 International - CC BY-NC 4.0. 


\section{LA RELIGION DANS LES DÉCISIONS RELATIVES À LA SANTÉ ET À L'ÉDUCATION DES ENFANTS EN DROIT CANADIEN ET OUÉBÉCOIS}

\section{José WOEHRLING}

Université de Montréal

\section{RÉSUMÉ}

Dans cette contribution, l'auteur examine les règles de droit canadien et québécois qui encadrent la prise en compte de la religion dans les décisions relatives à la santé et à l'éducation des enfants, décisions habituellement prises par les parents et parfois par les enfants eux-mêmes. Les lois adoptées dans ce domaine par le Parlement fédéral et les législatures provinciales, les lois québécoises faisant l'objet d'une attention particulière, peuvent être contestées comme ne respectant pas les droits constitutionnels des intéressés, tant les parents que les enfants. On évoquera donc les principales décisions de la Cour suprême du Canada dans lesquelles la Cour a été amenée à mettre en balance les droits des parents et ceux des enfants et à concilier le degré d'autonomie décisionnelle du mineur et la protection de sa vie et de sa santé.

\section{Abstract}

In this paper, the author examines the rules of Canadian and Quebec law governing the consideration of religion in decisions relating to health and education of children, decisions that are usually made by parents and sometimes by children themselves. The legislation adopted, in their respective fields, by the federal Parliament and the provincial legislatures, Quebec laws being accorded special attention, can be challenged as not respecting the constitutional rights of parents or children. We will thus consider the main decisions of the Supreme Court of Canada on the issue, in which the Court had to balance the rights of parents, those of children and reconcile the minors autonomy of decision-making and the protection of his/her life and health. 


\section{INTRODUCTION : LE CONTEXTE JURIDIQUE ET CONSTITUTIONNEL CANADIEN ET QUÉBÉCOIS}

Dans cette étude, notre objectif est d'examiner la prise en compte, par le droit canadien, de la religion dans l'encadrement des décisions relatives à la santé et à l'éducation des enfants, décisions habituellement prises par les parents ou les personnes qui en tiennent lieu, ou plus rarement par les enfants eux-mêmes. Les principales difficultés résident dans la nécessité de concilier les droits des parents et ceux des enfants tout en tenant compte de l'intérêt général de la société dans son ensemble, de donner un contenu concret au concept d'intérêt de l'enfant, qui constitue le concept juridique prédominant en la matière et, finalement, de déterminer le moment à partir duquel le mineur doit être habilité à faire ses propres choix.

En vertu de la Constitution canadienne de 1867, ce sont les entités fédérées, les provinces et territoires, qui ont la compétence générale sur le droit civil et l'état des personnes, ce qui comprend l'essentiel des questions relatives au droit de la famille ${ }^{1}$. Par ailleurs, le Parlement fédéral se voit attribuer plus spécialement la compétence sur le mariage et le divorce (la « célébration du mariage " étant à nouveau réservée aux législatures provinciales) ${ }^{2}$, ce qui veut dire que le Parlement fédéral peut seul légiférer sur la capacité au mariage et les conditions de fond de celui-ci, les provinces étant exclusivement compétentes pour régler les exigences formelles du mariage. Le Parlement fédéral légifère également sur le divorce et ses conséquences juridiques (pensions alimentaires, droit de garde et de visite, etc.) ${ }^{3}$. Cette situation entraîne une certaine complexité juridique : par exemple, dans une même province, les règles concernant les pensions alimentaires, le droit de garde et le droit de visite varient selon l'état matrimonial du couple qui se sépare, la loi fédérale s'appliquant si les parents divorcent, la loi provinciale s'ils mettent fin à une union civile ou à une union de fait. Par ailleurs, deux

1. Loi constitutionnelle de 1867, 30 \& 31 Vict., R.-U., c. 3 ; L.R.C. (1985), app. II, n 5, art. 91(13).

2. Loi constitutionnelle de 1867, art. 91(26) et 92(12).

3. Cette solution un peu curieuse s'explique pour des raisons historiques. En 1867, lors de l'adoption de la Constitution canadienne, les rédacteurs voulaient éviter que des considérations religieuses puissent influencer la législation en matière de divorce, en particulier au Québec dont la population était majoritairement catholique. En fait, on voulait s'assurer que la majorité catholique du Québec ne porterait pas atteinte au droit au divorce de la minorité protestante. 
traditions juridiques, la common law d'une part et le droit civil de tradition française de l'autre, coexistent au Canada. Au Québec, le droit familial est essentiellement contenu dans le Code civil québécois (et dans quelques lois plus spécifiques) alors qu'il relève encore largement, dans les autres provinces et dans les territoires, des règles de la common law (bien sûr complétées par les lois provinciales et parfois codifiées dans celles-ci) ${ }^{4}$.

Il ne sera évidemment pas possible de tracer ici un tableau complet du droit fédéral et de celui applicable dans toutes les entités fédérées. On se concentrera donc plutôt sur la situation au Québec, en donnant à l'occasion quelques détails sur le droit applicable dans d'autres provinces, dans la mesure où il sera tenu compte des principales décisions de la Cour suprême $\mathrm{du}$ Canada pertinentes sur le sujet, certaines portant sur le droit provincial du Québec, d'autres traitant de questions soulevées par le droit provincial applicable ailleurs au Canada.

Nous examinerons d'abord les questions que soulèvent les décisions concernant la santé des enfants (1), puis celles qui ont trait à leur éducation (2).

\section{LES DÉCISIONS RELATIVES À LA SANTÉ DES ENFANTS}

Avant l'atteinte par les enfants de leur majorité, ce sont normalement leurs parents, ou les personnes qui en tiennent lieu, qui prennent pour eux les décisions qui les concernent, y compris celles qui ont une portée ou une incidence religieuses. Au fur et à mesure que les enfants avancent en âge, ils acquièrent progressivement les capacités de jugement et la maturité nécessaires, d'abord pour exprimer leur opinion afin qu'il en soit tenu compte, ensuite pour faire leurs propres choix. C'est pourquoi divers régimes législatifs aménagent des paliers d'âge à partir desquels les enfants obtiennent le droit de prendre leurs propres décisions avant même d'atteindre leur majorité (1.1). Dans la conception et la mise en ouvre de ces régimes, la difficulté est évidemment de concilier deux objectifs qui sont, d'une part, la protection du mineur contre les mauvaises décisions qu'il pourrait prendre à son propre égard et, d'autre part, une certaine reconnaissance de son autonomie

4. Pour le droit familial au Québec, V. Castelli M. D. et Goubeau D., Précis du droit de la famille, Sainte-Foy, Presses de l'Université Laval, 2005. Pour le droit applicable dans les autres juridictions canadiennes, V. LAviolette N. et Audet J., L'essentiel du droit de la famille dans les provinces et territoires de common law au Canada, Cowansville, Éd. Yvon Blais, 2014. 
décisionnelle (1.2). Le domaine qui illustre probablement le mieux la tension entre ces deux objectifs est celui des décisions concernant la santé des enfants.

Comme pour le droit de la famille, la compétence de principe de légiférer dans le domaine des soins de santé appartient aux entités fédérées. La situation varie donc d'une province à l'autre. Néanmoins, le Parlement fédéral peut également intervenir dans ce domaine par l'entremise de sa compétence sur le droit criminel, dans la mesure où certains actes de nature médicale sont susceptibles de faire l'objet d'une réglementation pénale ${ }^{5}$.

\subsection{LE CONSENTEMENT DU MINEUR AUX SOINS MÉDICAUX ET LA PROTECTION DE SA VIE OU DE SA SANTÉ}

$\mathrm{Au}$ Québec, les dispositions législatives applicables prévoient que le consentement aux soins requis par l'état de santé d'une personne mineure est donné par le titulaire de l'autorité parentale ou par le tuteur. Cependant, à partir de quatorze ans, la personne mineure peut consentir seule à ces soins. Si son état exige qu'elle demeure dans un établissement de santé ou de services sociaux plus de douze heures, le titulaire de l'autorité parentale doit en être avisé, mais sans pour autant devoir être informé des motifs de la prise en charge ${ }^{6}$. On remarquera qu'un tel régime permet à une mineure d'obtenir un avortement ou une prescription de médicaments anticonceptionnels à partir de quatorze ans, sans implication des parents, ce qui peut s'avérer pertinent notamment dans les cas où ces derniers s'y opposeraient pour des raisons religieuses ou morales. De la même façon, les adolescents ont ainsi accès aux mesures de dépistage et de traitement des maladies sexuellement transmissibles sans que leurs parents soient nécessairement mis au courant. Si un mineur qui a atteint l'âge de quatorze ans refuse les soins

5. Par exemple, avant 1988, le Code criminel canadien interdisait l'avortement sauf pour des raisons thérapeutiques. Les dispositions en cause ayant été invalidées par la Cour suprême et n'ayant jamais été remplacées, l'avortement est maintenant possible sans restriction légale et relève essentiellement de la compétence provinciale sur les soins de santé.

6. Code civil du Québec, art. 14. La circoncision rituelle n'étant pas requise par l'état de santé des enfants sur lesquels elle est pratiquée, c'est l'article 18 du Code civil qui s'applique, en vertu duquel, là encore, le consentement doit être donné par le titulaire de l'autorité parentale dans le cas d'un mineur âgé de moins de quatorze ans. L'article ajoute que l'autorisation du tribunal est en outre nécessaire si les soins (non requis par l'état de santé du mineur) présentent un risque sérieux pour la santé ou s'ils peuvent causer des effets graves et permanents. En pratique, la circoncision n'est pas considérée comme ayant de tels effets et l'autorisation du tribunal n'est donc pas nécessaire. V. pour plus de détails ROBERT M.-P., « La circoncision rituelle au Canada », in ForTIER V. (dir.), La circoncision rituelle. Enjeux de droit, enjeux de vérité, Strasbourg, PUS, 2016, p. 302. 
nécessaires à sa santé, l'autorisation pourra être donnée par le tribunal ou, en cas d'urgence, lorsque sa vie est en danger ou son intégrité menacée, par le titulaire de l'autorité parentale ou le tuteur. Dans le cas d'un mineur de moins de quatorze ans, le refus injustifié des soins par le titulaire de l'autorité parentale ou le tuteur peut également être écarté par le tribunal, statuant dans le meilleur intérêt de l'enfant ${ }^{7}$. Dans les autres provinces canadiennes, des régimes similaires s'appliquent, soit en vertu de la loi, soit en vertu des règles de la common law applicables aux " mineurs matures ${ }^{8}$. Par exemple, la loi du Manitoba sur les services à l'enfant et à la famille fixe l'âge du consentement des mineurs à seize ans ${ }^{9}$.

Les tribunaux canadiens ont à plusieurs reprises autorisé des soins qui avaient été d'abord refusés par le titulaire de l'autorité parentale pour un motif d'ordre religieux. En 1995, la Cour suprême du Canada, dans une affaire provenant de l'Ontario ${ }^{10}$, a tranché la question de la conformité de telles interventions judiciaires au regard de la Charte canadienne des droits et libertés ${ }^{11}$. La Cour a jugé que celle-ci garantissait le droit des parents de prendre les décisions relatives à l'éducation et aux soins médicaux de leurs enfants en conformité avec leurs croyances religieuses, mais que les restrictions à ce droit des parents étaient justifiées si leur décision menaçait la vie, la sécurité ou la santé de l'enfant (l'affaire concernait une enfant de quelques semaines qui avait besoin d'une transfusion sanguine. Les parents, Témoins de Jéhovah, refusaient leur consentement) ${ }^{12}$.

En ce qui concerne les situations dans lesquelles c'était un adolescent qui refusait les soins de santé pour un motif religieux, les positions ont été plus nuancées. Certaines décisions appliquant la common law canadienne ont confirmé le refus, après que le tribunal ait constaté la maturité et l'aptitude du

7. Code civil du Québec, art. 16.

8. Sur cet aspect de la common law, V. Robertson G. B., « Children's Rights and Health Law: The "Mature Minor" Rule Revisited », in Anand S. (ed.), Children and the Law. Essays in Honour of Professor Nicholas Bala, Toronto, Irwin Law Inc., 2011, p. 39.

9. Loi sur les services à l'enfant et à la famille, C.P.L.M. chap. C80.

10. B. (R) c. Children's Aid Society of Metropolitan Toronto, [1995] 1 R.C.S. 315.

11. La Charte canadienne des droits et libertés (ci-après : la Charte canadienne) est contenue dans la partie I (articles 1 à 34) de la loi constitutionnelle de 1982, annexe B de la loi de 1982 sur le Canada, 1982, R.-U., c. 11 ; L.R.C. (1985), app. II, nº 44.

12. La Cour a analysé la question sous l'angle des articles 2(a) (liberté de religion) et 7 de la Charte canadienne des droits et libertés. Ce dernier article énonce : « Chacun a droit à la vie, à la liberté et à la sécurité de sa personne; il ne peut être porté atteinte à ce droit qu'en conformité avec les principes de justice fondamentale. » Les tribunaux canadiens font découler de l'article 7 un certain droit au respect de la vie privée et familiale, qui n'est pas reconnu expressément en tant que tel dans la Charte canadienne. 
mineur, alors que d'autres décisions ont plutôt autorisé les soins en dépit du refus du mineur ${ }^{13}$. On comprend qu'il est très difficile de systématiser cette jurisprudence dans la mesure où les décisions sont rendues en cherchant à concilier le degré d'autonomie décisionnelle du mineur et la protection de sa vie et de sa santé, cette conciliation étant forcément tributaire des faits de chaque espèce.

\subsection{AUTONOMIE DÉCISIONNELLE ET DEGRÉ DE MATURITÉ DU MINEUR}

Les régimes législatifs en vigueur peuvent être contestés si on estime qu'ils ne respectent pas les droits constitutionnels des personnes intéressées, parents ou enfants. C'est ce qui est arrivé dans le cas de la loi manitobaine mentionnée plus tôt, qui fixe à seize ans l'âge du consentement aux soins de santé. Selon cette loi, si l'enfant est âgé de seize ans et plus et refuse le traitement, son refus doit être respecté à condition qu'il comprenne l'information nécessaire à la décision à prendre et qu'il soit en mesure d'en évaluer les conséquences normalement prévisibles. Si l'enfant a moins de seize ans, la loi prévoit également qu'il faudra tenir compte de son opinion, mais celle-ci pourra être écartée par le tribunal dans le "meilleur intérêt de l'enfant ", même dans les cas où celui-ci est considéré comme apte à comprendre et à évaluer les conséquences de sa décision. La loi manitobaine a été contestée dans une affaire où une jeune fille âgée de quatorze ans et dix mois, appartenant aux Témoins de Jéhovah, avait refusé, avec l'accord de ses parents, une transfusion sanguine jugée médicalement nécessaire. Le tribunal saisi de l'affaire avait ordonné que la transfusion lui soit administrée d'autorité malgré le fait que les psychiatres ayant examiné l'intéressée aient été d'avis qu'elle comprenait les conséquences possibles d'un refus de traitement ${ }^{14}$.

Les droits constitutionnels invoqués au nom de la jeune fille pour contester la décision et la loi étaient le droit à l'égalité sans discrimination fondée sur l'âge, la liberté de religion et le droit à la liberté et à la sécurité de sa personne. La Cour suprême a rejeté cette contestation et validé la loi. La majorité des juges a considéré qu'une distinction basée sur l'âge du mineur est conforme aux réalités entourant le développement progressif des jeunes, le point de démarcation entre ceux âgés de seize ans et les mineurs de moins de seize ans étant rationnellement valable. À l'égard des mineurs de moins de seize ans, la difficulté inhérente dans l'évaluation de la «maturité » justifie que l'État

13. Pour une analyse plus détaillée de cette jurisprudence, V. OGILVIE M. H., Religious Institutions and the Law in Canada, $3^{\text {rd }}$ ed., Toronto, Irwin Law Inc., 2010, p. 384-387.

14. A.C. c. Manitoba (Directeur des services à l'enfant et à la famille), [2009] 2 R.C.S. 181. 
conserve le pouvoir suprême de décider s'il est réellement dans l'intérêt de l'enfant de lui permettre d'exercer son autonomie dans une situation donnée. Mais « l'intérêt de l'enfant» doit, à son tour, être interprété de manière à refléter et à respecter le droit croissant de l'adolescent à l'autonomie. Plus le tribunal est convaincu que l'enfant est capable de prendre lui-même des décisions de façon véritablement mature et indépendante, plus il doit accorder de poids à ses opinions dans l'exercice de son pouvoir discrétionnaire ${ }^{15}$. Autrement dit, la loi peut être considérée comme constitutionnelle à condition de l'interpréter comme permettant aux adolescents de moins de seize ans de démontrer qu'ils possèdent la maturité suffisante pour prendre les décisions médicales les concernant. La Cour a mentionné que, dans l'examen de la maturité d'un adolescent, le tribunal doit tenir compte des éléments suivants : la nature, le but et l'utilité du traitement médical recommandé, ainsi que ses risques et bénéfices; la capacité intellectuelle de l'adolescent et le discernement requis pour comprendre les renseignements qui lui permettraient de prendre la décision et d'en évaluer les conséquences possibles ; l'opinion de l'adolescent et la question de savoir si elle reflète véritablement ses valeurs et croyances profondes; l'impact que pourraient avoir le style de vie de l'adolescent, ses relations avec sa famille et ses affiliations sociales sur sa capacité d'exercer tout seul son jugement; l'existence de troubles émotionnels ou psychiatriques et les incidences de la maladie de l'adolescent sur sa capacité de décider. Enfin, il faut aussi prendre en considération le «patrimoine culturel, linguistique et religieux » de l'enfant.

Si une certaine autonomie décisionnelle est reconnue, en ce qui concerne les décisions concernant leur santé, aux mineurs qui ont atteint un âge ou un degré de maturité suffisant, on verra maintenant que cela n'est pas encore le cas pour ce qui est des décisions relatives à leur éducation.

15. «Plus le tribunal est convaincu que l'enfant est capable de prendre lui-même des décisions de façon mature et indépendante, plus il accordera de poids à ses opinions dans l'exercice de son pouvoir discrétionnaire [...]. Si, après une analyse approfondie et complexe de la capacité de la jeune personne d'exercer son jugement de façon mature et indépendante, le tribunal est convaincu qu'elle a la maturité nécessaire, il s'ensuit nécessairement, à mon avis, qu'il faut respecter ses opinions. Il ressort d'une telle approche qu'en matière de traitement médical, les moins de 16 ans devraient avoir le droit de tenter de démontrer que leur opinion sur une décision touchant un traitement médical particulier révèle une indépendance d'esprit et une maturité suffisantes. » (A.C. c. Manitoba, § 87 ; juge Abella). 


\section{LES DÉCISIONS RELATIVES À L'ÉDUCATION DES ENFANTS}

Après avoir examiné les restrictions relatives à l'éducation et aux pratiques religieuses susceptibles d'être imposées au droit de visite des parents en cas de séparation (2.1), nous verrons selon quelles modalités les parents peuvent choisir l'école privée ou l'éducation à domicile, plutôt que l'école publique, notamment pour des raisons religieuses (2.2) et quels sont les accommodements et exemptions susceptibles d'être réclamés au nom de la religion dans le contexte de l'éducation des enfants (2.3). Nous terminerons en soulevant la question du droit des adolescents de prendre eux-mêmes certaines décisions relatives à leur éducation (2.4).

\subsection{LES RESTRICTIONS RELATIVES À L'ÉDUCATION RELIGIEUSE SUSCEPTIBLES D'ÊTRE IMPOSÉES DANS L'INTÉRÊT DE L'ENFANT}

Les parents séparés conservent à l'égard de leurs enfants les droits et devoirs d'éducation, de garde et de surveillance, mais la situation de séparation amène presque toujours un démembrement de l'autorité parentale ${ }^{16}$. Lorsque la garde des enfants est confiée à l'un des parents, comme c'est le plus souvent le cas, l'autre conserve ordinairement un droit de visite et de sortie (ou droit d'accès, selon la loi fédérale sur le divorce). En effet, tant la loi fédérale que le droit civil québécois posent le principe selon lequel l'enfant doit avoir avec chacun de ses parents le maximum de contacts compatible avec son propre intérêt. Dans ce contexte, les tribunaux sont habilités à imposer des restrictions aux droits de visite et de sortie octroyés à un parent lorsqu'ils estiment ces restrictions nécessaires dans l'intérêt de l'enfant. Des restrictions relatives à l'instruction religieuse de l'enfant ou à sa participation à des activités de culte ou de démarchage sont parfois imposées, notamment dans le cas de parents qui font partie des Témoins de Jéhovah.

En 1993, la Cour suprême du Canada a rendu deux décisions le même jour sur cette question, en appel l'une de la Cour d'appel du Québec ${ }^{17}$,

16. En cas de conflit entre les parents sur une décision concernant l'enfant, par exemple en ce qui concerne le choix de la religion ou l'éducation religieuse, chacun d'entre eux peut saisir le tribunal (la Cour supérieure) qui devra statuer dans l'intérêt de l'enfant après avoir favorisé la conciliation entre les parties (art. 604 du Code civil du Québec). La décision du tribunal met fin au conflit entre les parents, mais elle n'entraîne aucune perte d'autorité parentale. Le même mécanisme pourrait également servir à trancher un différend entre un enfant et ses parents.

17. P. (D.) C. S. (C.), [1993] 4 R.C.S. 141. 
l'autre de la Cour d'appel de la Colombie britannique ${ }^{18}$. Dans chacune des deux affaires, le parent non-gardien, le père en l'occurrence, appartenait aux Témoins de Jéhovah et contestait les restrictions relatives à la pratique religieuse dont son droit de visite avait été assorti, en invoquant sa liberté de religion garantie par la Constitution. La Cour a reconnu que les restrictions en cause constituaient effectivement une limite à cette liberté, mais qu'une telle limite serait justifiée par la recherche du meilleur intérêt de l'enfant dans les cas où il apparaîtrait que la participation à des pratiques religieuses entraîne des effets nocifs pour ce dernier.

Une tendance qu'on constate dans la jurisprudence consiste à mettre l'accent sur l'intégration de l'enfant à la fois dans son milieu familial et dans le milieu social plus large auquel il appartient, ce qui est susceptible de défavoriser les parents appartenant à un groupe religieux qui prône l'isolement d'avec la société et d'avec les membres de la famille qui ne partagent pas les mêmes convictions, comme c'est le cas par exemple des Témoins de Jéhovah $^{19}$. Par ailleurs, les tribunaux expriment d'habitude leur préférence pour les solutions qui restreignent aussi peu que possible la capacité de chaque parent de partager ses convictions religieuses avec ses enfants. Ils soulignent qu'il est dans l'intérêt des enfants de connaître le plus pleinement possible le parent non-gardien, y compris sous l'angle de ses croyances et de sa pratique religieuses ${ }^{20}$.

\subsection{LE CHOIX DE L'ÉCOLE PRIVÉE OU DE L'ÉDUCATION À DOMICILE}

Le droit à la liberté reconnu par l'article 7 de la Charte canadienne a été interprété comme incluant le droit des parents de prendre les décisions concernant l'éducation de leurs enfants. Cela comprend notamment le droit de choisir de les éduquer à la maison plutôt que de les envoyer à l'école, ainsi que le droit de choisir une école privée plutôt que l'école publique. Lorsque la décision de les éduquer à domicile repose sur des raisons religieuses, ou que les parents choisissent une école privée religieuse,

18. Young c. Young, [1993] 4 R.C.S. 3.

19. En ce sens, V. Ogilvie M. H., op. cit. p. 373.

20. « Ni les divergences d'opinions des parents sur les questions religieuses ni la franche discussion avec les enfants des perceptions religieuses des deux parents ne seront automatiquement préjudiciables. En fait, elles peuvent souvent être avantageuses [...]. L'intérêt de l'enfant englobe certainement une discussion franche sur la croyance religieuse, par opposition à l'endoctrinement, au recrutement ou au harcèlement qui a pour objectif de miner la décision du parent qui a la garde sur la question de la religion » (P. (D.) c. S. (C.), précit., p. 191 ; juges Cory et Iacobucci). 
on pourra également invoquer la liberté de religion. Dans une décision de $1986^{21}$, la Cour suprême a jugé que la liberté de religion n'empêchait pas l'État d'exiger que les parents qui veulent éduquer leurs enfants à domicile soient tenus de faire une demande d'exemption et de se soumettre à des inspections par le ministère provincial de l'Éducation. De la même façon, les écoles privées doivent respecter les normes pédagogiques définies par l'État provincial pour que les certificats qu'elles délivrent soient reconnus et, a fortiori, pour obtenir des subventions publiques. Cependant, comme on le verra plus loin, la Cour suprême a mis des limites aux directives que le ministère provincial de l'Éducation peut imposer à une école privée religieuse en ce qui concerne l'enseignement de la religion dont l'école se réclame.

Par ailleurs, la Constitution canadienne a été interprétée comme ne reconnaissant aucun droit au subventionnement public des écoles privées, religieuses ou autres, même si les parents qui y envoient leurs enfants sont de ce fait assujettis à une double charge financière puisqu'ils continuent de devoir payer l'impôt scolaire destiné aux écoles publiques ${ }^{22}$. Néanmoins, sans être constitutionnellement nécessaires, les subventions aux écoles privées religieuses ne sont pas constitutionnellement prohibées (comme c'est le cas aux États-Unis). Et de fait, un certain nombre de provinces canadiennes, dont le Québec, subventionnent les écoles privées, dans le cas du Québec à hauteur de $60 \%$ de ce que l'État dépense par enfant dans le système public. D'autres provinces par contre, comme l'Ontario (la province la plus peuplée), refusent un tel subventionnement.

La fréquentation des écoles privées religieuses a sensiblement augmenté depuis une quinzaine d'années, notamment, semble-t-il, à la suite de la laïcisation imposée aux écoles publiques par les tribunaux en application de la Charte canadienne des droits et libertés. En effet, avant l'entrée en vigueur de celle-ci, en 1982, un enseignement religieux et certaines pratiques religieuses (par exemple la prière au début de la journée scolaire) existaient dans les écoles publiques de la plupart des provinces. Tant l'enseignement religieux que les pratiques religieuses à l'école publique ont été déclarées inconstitutionnelles, comme étant incompatibles avec l'obligation de neutralité religieuse de l'État et la liberté de conscience et de religion, et ceci même si les parents pouvaient se prévaloir d'un droit d'exemption pour leurs enfants. En effet, les tribunaux ont considéré que le fait de réclamer

21. La Reine c. Jones, [1986] 2 R.C.S. 284.

22. Adler c. Ontario, [1996] 3 R.C.S. 609. 
le bénéfice de l'exemption pourrait avoir pour effet de stigmatiser et de singulariser les enfants concernés ${ }^{23}$.

La neutralité religieuse de l'État (ou laïcité) n'est pas expressément proclamée dans la Constitution canadienne, mais les tribunaux ont jugé qu'une obligation de neutralité religieuse s'imposant à l'État constitue un élément implicite de la liberté de conscience et de religion ${ }^{24}$. Tenant compte de son caractère implicite, et d'autres particularités de la Constitution, ce principe de neutralité est interprété de façon moins rigoureuse qu'aux États-Unis (où il figure explicitement au Premier Amendement). Ainsi, comme on vient de le voir, le principe de neutralité n'a pas été jugé incompatible avec le financement public des écoles religieuses, à condition qu'un tel financement ne soit pas discriminatoire. De la même façon, non seulement le principe de neutralité religieuse n'interdit pas les " accommodements » en faveur de la religion, mais au contraire, il les rend obligatoires dans certains cas, bien que sous un autre angle, on pourrait également les considérer comme une façon d'aider et de favoriser la religion dans la mesure où ils ont pour but de supprimer certains obstacles à la pratique religieuse.

\subsection{LES ACCOMMODEMENTS RELIGIEUX SUSCEPTIBLES D'ÊTRE RÉCLAMÉS EN MATIÈRE D'ÉDUCATION}

En droit canadien, l'accommodement (ou aménagement) raisonnable fondé sur la religion consiste à modifier ou à aménager une règle d'application générale pour faciliter la pratique religieuse, lorsque la règle générale constitue un empêchement ou un obstacle à cette pratique. L'obligation d'accommodement est considérée comme découlant principalement du droit à l'égalité, car les tribunaux estiment qu'une règle générale qui crée des inconvénients pour

23. Zylberberg c. Sudbury Board of Education, (1988) 65 O.R. (2d) 641 (C.A. Ont.), autorisation d'appel en Cour suprême refusée; Canadian Civil Liberties Association c. Ontario (Minister of Education), (1990) 71 O.R. (2d) 341 ; 65 D.L.R. (4th) 1 (C.A. Ont.), autorisation d'appel en Cour suprême refusée.

24. Sur le principe de neutralité religieuse de l'État en droit canadien, V. JUKIER R. et WoenRLING J., "Religion and the Secular State. National Report for Canada », in MARTinez Torron J. et Durham W. C. (eds), Religion and the Secular State. National Reports (XVIIIth Congress of the International Academy of Comparative Law), Madrid, Facultad de Derecho de la Universidad Complutense, 2015, p. 155. - Woenrling J., "Quelle place pour la religion dans les institutions publiques? », in Gaudreault-DesBiens J.-F. (dir.), Le droit, la religion et le "raisonnable ", Montréal, Thémis, 2009, p. 115. - Moon R., "Freedom of Religion Under the Charter of Rights: The Limits of State Neutrality », University of British Columbia Law Review, (2012) 45, p. 497. - BoutoubA N. et Bernatchez S., " L'État et la diversité religieuse au Canada : une possible histoire du principe de neutralité de l'État », Revue de droit de l'Université de Sherbrooke, numéro spécial, 2013, p. 9. 
un groupe particulier, alors qu'elle est favorable ou neutre à l'égard de la majorité, constitue une discrimination indirecte à l'égard de ce groupe. Dans le cas des accommodements religieux, l'obligation est également fondée sur la liberté de conscience et de religion ${ }^{25}$.

C'est ainsi que la Cour suprême du Canada a décidé, dans une affaire datant de $2006^{26}$, qu'une école publique de Montréal était tenue d'autoriser un élève de religion sikh à conserver son kirpan (un poignard de métal rituel) durant sa présence à l'école, le faisant ainsi bénéficier d'une exemption d'application du règlement général de l'école interdisant la possession d'armes par les élèves. Elle a souligné qu'on n'avait jamais rapporté d'incident impliquant un kirpan dans une école et que l'intéressé avait accepté de porter le kirpan dans une gaine solidement fermée et cousue sous ses vêtements. Il existe également des décisions autorisant les enseignants d'une école publique de l'Ontario à porter le kirpan à l'école ${ }^{27}$. Par ailleurs, d'autres décisions judiciaires ont interdit le port du kirpan dans les palais de justice et à bord des avions ${ }^{28}$. Des décisions judiciaires ont également reconnu

25. Sur l'accommodement raisonnable en droit canadien, V. WoeHrLing J., « Aménagement de la diversité religieuse et conflits entre droits fondamentaux. Le contexte juridique canadien », in BRIBOSIA E. et RORIVE I. (dir.), L'accommodement de la diversité religieuse. Regards croisés - Canada, Europe, Belgique, Bruxelles, Peter Lang, 2015, p. 135. - BOSSET P., « Les fondements juridiques et l'évolution de l'obligation d'accommodement raisonnable ", in JÉZÉQUel M. (dir.), Les accommodements raisonnables : quoi, comment, jusqu'où ? Cowansville, Éd. Yvon Blais, 2007, p. 3.

26. Multani c. Commission scolaire Marguerite-Bourgeoys, [2006] 1 R.C.S. 256.

27. Pandori c. Peel Board of Education, (1990) 12 C.H.R.R. D/364 (Commission d'enquête, Ontario). Il s'agissait de deux plaintes conjointes relatives au port du kirpan par un professeur et par des élèves sikhs à l'école. Le professeur et les élèves se voyaient opposer une résolution de la Commission scolaire de Peel interdisant le port du kirpan dans l'enceinte de l'école. Une commission d'enquête établie en vertu du Code ontarien des droits de la personne est arrivée à la conclusion, au vu de la preuve, que le port du kirpan devait être autorisé dans cette école, tant pour les élèves que pour les enseignants et les membres de l'administration, mais aux conditions qu'il soit d'une taille raisonnable, porté en dessous des habits de façon à être invisible et maintenu de façon assez ferme dans sa gaine pour être difficile, mais pas impossible, à en sortir. La décision de la commission devait être confirmée par la Cour divisionnaire d'Ontario : Peel Board of Education c. Ontario Human Rights Commission, (1991) 3 O.R. (3d) 351 (Cour divisionnaire, Ontario). Autorisation d'appel en cour d'appel rejetée le 12 août 1991.

28. Dans Hothi c. R., [1985] 3 W.W.R. 256 (Cour du Banc de la Reine du Manitoba) confirmé à [1986] 3 W.W.R. 671 (Cour d'appel du Manitoba), la Cour du Banc de la Reine du Manitoba a confirmé l'ordonnance d'un juge de la Cour provinciale interdisant le port du kirpan dans une salle d'audience. Dans le même sens, V. R. c. Kaur, [1997] Q.J. No. 5066 (cour municipale de Ville Saint-Laurent). Dans Nijjar c. Lignes aériennes Canada 3000 Ltée, un tribunal canadien des droits de la personne, constitué en vertu de la loi canadienne sur les droits de la personne, a rejeté la plainte d'un sikh qui s'était vu refuser l'accès à un avion à cause de son kirpan, bien qu'il s'agissait en l'occurrence, 
que les écoles publiques ont l'obligation d'autoriser les absences des élèves (et des enseignants) pour fêtes religieuses ${ }^{29}$. D'autres accommodements ont été négociés, sans que l'intervention d'un tribunal soit nécessaire, comme celui autorisant les jeunes filles à porter un pantalon long pour les cours de gymnastique, à la place du short, de façon à permettre le respect des règles de modestie de l'islam ${ }^{30}$.

L'obligation d'accommodement a par ailleurs des limites, puisque l'accommodement ne doit être accordé que dans la mesure où il est " raisonnable », ce qui signifie qu'il peut être légitimement refusé s'il entraîne une « contrainte excessive ». Dans le cas d'un accommodement réclamé auprès d'une école publique, il y aura contrainte excessive si l'accommodement entraîne des coûts exagérés ou une entrave importante au fonctionnement de l'école. L'accommodement devra également être refusé s'il entraîne une atteinte aux droits ou aux libertés d'autrui.

Selon la même logique, existe-t-il un droit des parents d'obtenir que leurs enfants soient exemptés de certains éléments du programme obligatoire des écoles publiques, en particulier lorsque les parents s'y opposent pour des raisons religieuses ${ }^{31}$ ? La question se pose notamment pour les cours d'éducation sexuelle. Le gouvernement de l'Ontario, qui a mis en

selon le plaignant, d'un «kirpan de voyage» dont la lame ne dépassait pas 4 pouces de longueur. Le tribunal a tenu compte de l'environnement particulier d'un avion, où il n'est possible d'avoir accès ni à des services médicaux d'urgence ni à une assistance policière. Par contre, dans Pritam Singh c. Workmen's Compensation Board Hospital, (1981) 2 C.H.R.R. D/459 (Commission d'enquête, Ontario), M. Singh avait été informé qu'il ne pourrait pas passer de tests à l'hôpital s'il n'ôtait pas son kirpan, ce qu'il avait refusé de faire; la commission d'enquête décide que l'hôpital aurait pu trouver une solution d'accommodement respectant les croyances de M. Singh; elle ordonne qu'à l'avenir les patients de religion sikh soient autorisés à conserver leur kirpan, à condition qu'il soit d'une longueur raisonnable, pendant qu'ils reçoivent des soins à l'hôpital.

29. Islamic Schools Federation of Ontario c. Ottawa Board of Education, (1997) 145 D.L.R. (4th) 659 (Cour divisionnaire de l'Ontario); Commission scolaire régionale de Chambly c. Bergevin, [1994] 2 R.C.S. 525 (Cour suprême du Canada).

30. Pour plus de détails, V. Woenrling J., « La place de la religion dans les écoles publiques du Québec », Revue juridique Thémis, (2007) 41, p. 651.

31. Sur cette question des exemptions religieuses au curriculum obligatoire des écoles publiques, telle qu'elle a été abordée par les tribunaux des États-Unis et par les organes d'application de certaines conventions internationales sur les droits de la personne, V. Woenrling J., «Liberté de religion et demandes d'exemption à l'égard du curriculum scolaire obligatoire : les réponses des tribunaux américains », in LEFEBVRE B. et Leduc A. (dir.), Mélanges Pierre Ciotola, Montréal, Thémis, 2012, p. 563 ; Woenrling J., « L'enseignement des religions et de l'éthique à l'école publique : les principes dégagés par la Cour européenne des droits de l'homme et le Comité des droits de l'homme des Nations unies (affaires Folgero, Zengin et Leirvag) », Revue québécoise de droit international, hors-série juin 2015, p. 359. 
place un nouveau programme de ce genre l'année dernière, a dû reculer devant l'ampleur des protestations, particulièrement celles venant des parents catholiques et musulmans, et a accepté que les parents puissent retirer leurs enfants du programme après avoir dans un premier temps exclu cette possibilité. Le gouvernement québécois lance lui aussi cette année un nouveau programme-pilote d'éducation sexuelle et, pour l'instant, il a annoncé qu'il ne permettrait aucune exemption.

La question des exemptions religieuses au programme obligatoire des écoles publiques a fait l'objet d'une décision de la Cour suprême du Canada en 2012. Jusqu'en 2008, le programme des écoles publiques québécoises comprenait un enseignement religieux catholique ou protestant et un enseignement moral, les parents devant obligatoirement choisir entre les deux. L'enseignement religieux ayant été déclaré inconstitutionnel par les tribunaux $^{32}$, le gouvernement québécois a décidé d'introduire une nouvelle formule à partir de 2008, celle d'un cours unique d'éthique et de culture religieuse obligatoire pour tous les élèves (communément connu comme le cours ECR). Le volet éthique du programme vise à encourager les élèves à porter un regard critique sur leur propre conduite éthique et sur celle d'autrui, ainsi que sur les valeurs et les normes adoptées par différents groupes religieux. La partie du cours consacrée à la culture religieuse est destinée à présenter de façon neutre et objective les diverses traditions religieuses du monde, en insistant sur celles traditionnellement présentes au Québec. Le programme exige des enseignants qu'ils fassent preuve d'objectivité et d'impartialité. Ils ne doivent pas affirmer la vérité d'un système particulier de croyances ou tenter d'influencer les convictions de leurs élèves, mais favoriser la connaissance d'une variété de valeurs, de convictions et de cultures. Le cours ECR est obligatoire au primaire et au secondaire, sans possibilité d'exemption, dans les écoles publiques et dans les écoles privées subventionnées par le gouvernement, y compris les écoles privées religieuses.

Depuis son entrée en vigueur, le programme ECR fait l'objet de critiques provenant de deux camps opposés. D'une part, ceux qui pensent que sous couvert de neutralité et de visée culturelle, le cours comporte des éléments d'endoctrinement contraires aux principes d'un enseignement public laique. Ils réclament l'abolition pure et simple du volet « culture religieuse ». D'autre part, le cours ECR est également critiqué par les parents animés de fortes convictions religieuses qui considèrent que l'enseignement en question, précisément à cause de son objectif de neutralité, véhicule un relativisme qui

32. V. supra, note 23 et les développements du texte. 
entre en conflit avec leurs efforts pour transmettre leurs propres convictions à leurs enfants. Ces parents, quant à eux, réclament pour leurs enfants le bénéfice d'une exemption du cours, ce qui leur a été refusé jusqu'à présent. Ils se sont donc pourvus devant les tribunaux. La Cour suprême a rendu son jugement dans cette affaire en $2012^{33}$.

Malheureusement, le jugement ne va pas au fond des choses, le contexte factuel de la décision ne l'ayant pas permis. En effet, les requérants ont contesté le cours ECR de façon prématurée, très peu de temps après sa mise en application, ces circonstances faisant en sorte qu'il était très difficile pour la Cour d'évaluer les effets réels du programme. Sur la base des éléments incomplets qui lui étaient soumis, à savoir le seul cadre pédagogique du cours, la Cour suprême a conclu que les requérants n'avaient pas réussi à faire la preuve que le nouveau programme enfreignait leur liberté de religion ou celle de leurs enfants. Néanmoins, dans la mesure où elle s'est prononcée sur les questions de fond, la Cour a jugé que le fait d'exposer les enfants à une présentation globale des diverses religions, sans les obliger à y adhérer, ne constituait pas un "endoctrinement» des élèves. Elle a également conclu que l'exposition précoce des enfants à des réalités autres que celles qu'ils vivent dans leur environnement familial immédiat constitue un fait de la vie en société et que suggérer que le fait même d'exposer des enfants à différents faits religieux porterait atteinte à leur liberté de religion ou à celle de leurs parents reviendrait à rejeter la réalité multiculturelle de la société canadienne et méconnaître les obligations de l'État québécois en matière d'éducation publique. Par ailleurs, certains des juges ont souligné que même si le programme ne portait pas en lui-même atteinte à la liberté de religion, une telle atteinte pourrait éventuellement résulter de sa mise en application concrète, ce qui suggère que la question pourrait être portée à nouveau devant les tribunaux à l'avenir.

En fait, la Cour suprême a eu l'occasion de se pencher à nouveau sur le cours ECR dès 2015, mais sous un autre angle. En effet, il s'agissait cette fois du recours d'un collège catholique privé subventionné, donc obligé d'enseigner le cours ECR, et qui réclamait le droit de l'enseigner, dans tous ses aspects, avec une orientation catholique plutôt que l'orientation neutre et non confessionnelle imposée par le ministère. La Cour a effectivement jugé que le fait d'imposer à un collège catholique l'obligation d'enseigner le catholicisme avec une orientation neutre et laïque entraînait une atteinte non justifiable à la liberté de religion. Par contre, selon la Cour, le ministère 
était justifié d'exiger du collège qu'il adopte l'orientation neutre et laïque pour enseigner les traditions religieuses autres que le catholicisme ainsi que l'éthique, y compris celle des autres religions ${ }^{34}$. Et la Cour ajoute que, dans le cas d'une école secondaire confessionnelle, où les élèves acquièrent des connaissances au sujet des préceptes d'une religion particulière pendant toute la durée de leurs études, il est encore plus important qu'ils explorent, de façon aussi objective que possible, d'autres systèmes de croyances ainsi que leurs fondements ${ }^{35}$.

\subsection{LA SITUATION DES ADOLESCENTS CONCERNANT LES CHOIX RELATIFS À LEUR ÉDUCATION}

Pour les jeunes enfants, ce sont les parents qui font les choix relatifs à l'éducation et à la formation religieuse et morale. Mais qu'en est-il des adolescents ? Devrait-on prévoir en matière de choix éducatifs les mêmes modalités qu'en matière de choix portant sur les soins de santé, c'est-à-dire un âge à partir duquel le mineur est habilité à faire ses propres choix, même s'ils l'amènent à prendre d'autres positions que ses parents ? À l'époque où le choix existait entre l'enseignement religieux et l'enseignement moral dans les écoles publiques du Québec, la loi prévoyait que c'est à partir de la troisième année du secondaire, donc normalement vers quatorze ans, que l'élève pouvait effectuer lui-même ce choix. Le législateur lui reconnaissait donc la capacité d'exercer directement en ce domaine sa liberté de conscience et de religion. Par contre, à l'heure actuelle, seul un élève majeur, ou dans le cas d'un élève mineur les parents de celui-ci, peuvent demander une exemption de l'application du régime pédagogique « pour des raisons humanitaires ou pour éviter un grave préjudice à l'élève ${ }^{36}$. Il n'est pas impossible d'imaginer que l'on puisse se fonder sur les droits constitutionnels, la liberté de conscience et de religion et le droit à l'égalité en particulier, pour contester une situation dans laquelle les adolescents ne se voient pas reconnaître le droit de choisir eux-mêmes certaines modalités de leur éducation morale et religieuse. D’ores et déjà en droit québécois, en considérant les différentes limites d'âge prévues par le Code civil et par les lois particulières, on constate que l'âge de quatorze ans apparaît comme une étape significative dans le parcours vers la majorité. Si à l'avenir le législateur québécois décidait d'établir une possibilité d'exemption du cours ECR, il conviendrait donc probablement

34. École secondaire Loyola c. Québec (Procureur général), [2015] 1 R.C.S. 613.

35. Ibid., \& 72 (juge Abella).

36. Loi sur l'instruction publique, L.R.Q., ch. I-13.3, art. 222, al. 2. 
qu'il prévoie le même régime que celui qui s'appliquait anciennement au choix entre l'enseignement moral et l'enseignement religieux, c'est-à-dire de permettre à l'élève de demander lui-même l'exemption à partir de la troisième année du secondaire.

\section{CONCLUSION : L'INTÉRÊT DE L'ENFANT, UN CONCEPT POLYMORPHE}

Le concept juridique prédominant en matière de relations parents-enfants et de décisions relatives aux enfants est celui de l'intérêt de l'enfant. En vertu du Code civil du Québec, l'autorité parentale doit s'exercer dans l'intérêt de l'enfant et dans le respect de ses droits ${ }^{37}$. La loi fédérale sur le divorce prévoit que les juges doivent tenir compte du meilleur intérêt de l'enfant dans leurs décisions au sujet des ententes parentales à l'issue d'un divorce. La Convention relative aux droits de l'enfant des Nations unies dispose à son article 3 que «dans toutes les décisions qui concernent les enfants, qu'elles soient le fait des institutions publiques ou privées de protection sociale, des tribunaux, des autorités administratives ou des organes législatifs, l'intérêt supérieur de l'enfant doit être une considération primordiale ».

Ce concept d'intérêt de l'enfant est appliqué différemment selon que l'enfant concerné a ou non atteint une maturité suffisante. Comme nous l'avons vu précédemment, en matière de consentement aux soins de santé, la Cour suprême du Canada considère qu'il y a en quelque sorte une présomption voulant que l'intérêt d'un enfant ayant atteint une maturité suffisante suppose qu'on reconnaisse son autonomie décisionnelle (il y a donc pour ainsi dire «fusion» entre l'intérêt du mineur et le respect de son autonomie). Avec le consentement aux soins, il s'agit d'une situation où l'évaluation individualisée du degré de maturité du mineur est à la fois nécessaire et possible. Ce n'est évidemment pas toujours le cas. Par exemple, en matière de décision concernant l'enseignement religieux ou moral, une évaluation individualisée serait probablement trop complexe sur le plan administratif. Il faut donc laisser la possibilité au législateur de fixer les étapes qui ponctuent l'acquisition de certains éléments d'autonomie décisionnelle pour les mineurs. Ces seuils législatifs possèdent inévitablement une part d'arbitraire. Le cas échéant, ils pourront être contestés sur la base des droits constitutionnels des mineurs,

37. Code civil du Québec, art. 33 : «Les décisions concernant l'enfant doivent être prises dans son intérêt et dans le respect de ses droits. Sont pris en considération, outre les besoins moraux, intellectuels, affectifs et physiques de l'enfant, son âge, sa santé, son caractère, son milieu familial et les autres aspects de sa situation. » 
notamment leur droit à l'égalité. Quant aux mineurs qui n'ont pas atteint la maturité suffisante pour prendre leurs propres décisions, ils ont essentiellement le droit à des décisions prises pour eux par d'autres dans leur meilleur intérêt : leurs parents en situation habituelle, sinon les organismes de protection de l'enfance ou encore les tribunaux qui doivent arbitrer les conflits des parents entre eux, ceux des parents avec les organismes étatiques ou encore, dans certains cas, les conflits entre les parents et les enfants eux-mêmes.

Le consensus dominant dans notre société semble être que le meilleur intérêt de l'enfant passe par la maximisation de ses potentialités de développement vers l'autonomie personnelle et l'épanouissement individuel (son « autoréalisation »). C'est là un point de vue qui correspond bien au libéralisme individualiste, qui sert de soubassement idéologique aux instruments de protection des droits de l'homme au Canada et ailleurs. On comprend qu'un tel point de vue entrera en conflit avec des conceptions plus communautaristes selon lesquelles ce sont le maintien de l'appartenance des individus au groupe, notamment religieux, et la cohésion de celui-ci qui doivent être recherchés en priorité. Par ailleurs, la prédominance conférée à l'intérêt de l'enfant à l'autoréalisation ne fait pas disparaître toutes les difficultés, ce critère étant lui-même susceptible d'appréciations et d'applications diverses. Par exemple, si l'on revient à la problématique du cours d'éthique et de culture religieuse et à la critique que lui adressent les parents animés de fortes convictions religieuses, il reste encore à déterminer quel serait le facteur le plus nuisible, immédiatement ou à plus long terme, pour l'autoréalisation des enfants : le conflit d'allégeances susceptible de ternir leurs relations avec leurs parents ou le fait d'être privé de l'exposition aux idées et informations considérées comme nécessaires pour développer leur esprit critique et leur tolérance envers les différences culturelles et religieuses (et le fait d'être privés d'une interaction significative avec leurs pairs dans cet important domaine) ? Si l'on en revient par ailleurs à l'intérêt de l'État, celui-ci a un intérêt légitime, selon la Cour suprême du Canada, à s'assurer que les élèves de toutes les écoles (privées autant que publiques) seront en mesure, une fois devenus adultes, de se comporter avec ouverture et respect lorsqu'ils devront faire face aux différences culturelles et religieuses. Une démocratie multiculturelle dynamique doit pouvoir compter sur la capacité de ses citoyens de discuter de manière réfléchie et ouverte ${ }^{38}$.

38. École secondaire Loyola c. Québec, précit., § 48 (juge Abella). 